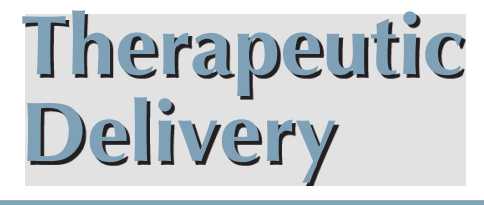

\title{
Diabetes management strategies: can nanoparticles be used to therapeutically deliver insulin?
}

\section{...this new strategy for oral insulin that targets the colon seems to pave a new way in oral delivery of insulin."}

First draft submitted: 22 November 2016; Accepted for publication: 25 November 2016; Published online: 16 January 2017

\section{Keywords: diabetes $\bullet$ nanotechnology $\bullet$ oral delivery}

Langerhans $\beta$ cells of the pancreas secrete insulin, which is a $60 \mathrm{kDa}$ peptide with a pivotal role in the regulation of carbohydrate metabolism [1]. In patients with Type 1 or Type 2 diabetes mellitus, low levels of insulin, predominantly owing to destruction of the pancreatic $\beta$ cells and/or a progressive insulin secretory defect, lead to an increase in gluconeogenesis and glycolysis in the liver and result in increased glucose levels in blood and urine [2]. A goal of insulin therapy in patients with diabetes is to simulate normal insulin secretion restoring glycemic control without leading to hypoglycemia. There are two main methods that aim to simulate normal insulin secretion: the first is the continuous subcutaneous insulin infusion with the implant of a small pump, while the alternative and more adopted strategy is the multiple daily injections, which uses a combination of long-acting insulin to provide basal insulin replacement, with separate injections of a rapid-acting insulin at mealtimes [1,3]. New formulations of long-term insulin are constantly on the development to increase the duration of the therapeutic efficacy and minimize patient's discomfort. However, all these therapeutic strategies use the parenteral administration route, which implies patient's pain, significant reduction in quality of life and, sometimes, poor patient adherence to therapy [4].

Therefore, several research groups have explored alternative routes of administration focusing their attention on new insulin formulations able to avoid invasive administration [5], including ocular, vaginal, rectal, oral, buccal, sublingual, nasal and others [6]. Among these, the oral formulation of insulin seems to be able to revolutionize the management of insulin-dependent diabetic patients, thanks to its larger absorption surface area and easy administration [6]. However, the main challenge in the development of oral insulin resides in its low bioavailability. Indeed, there are different hurdles in insulin oral delivery:

- It can be easily degraded by the acidic conditions in stomach and by the proteases in the GI tract;

- It is hydrophilic so it has a limited permeation through the intestinal epithelium since it is retained in the mucus;

- It has been absorbed by the portal vein so it has been first delivered to the liver.

Therefore, the liver first-pass effect determines a dramatic reduction in bioavailability [7]. Moreover, also variations in peristalsis and, hence, in intestinal transit and stool consistency determine variations that should be taken into account.

Nanotechnology has made several efforts to solve these limitations and a lot of different strategies have been exploited using natural polymers, such as chitosan, alginate,

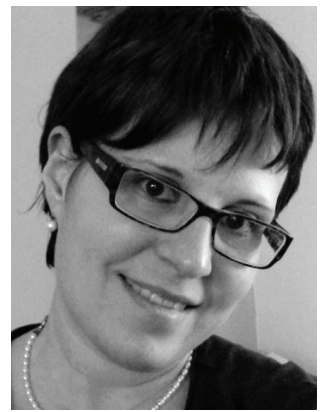

Serena Mazzucchelli Department of Biomedical \& Clinical Sciences 'L Sacco', University of Milan, via GB Grassi, 74, 20157 Milan, Italy *Author for correspondence: Tel. +3902390440450 serena.mazzucchelli@gmail.com

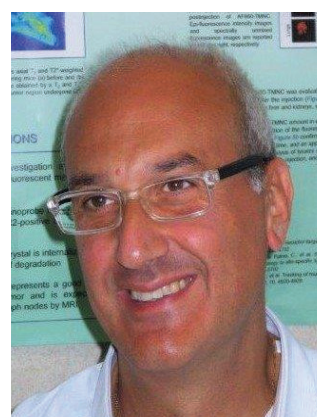

Fabio Corsi ${ }^{1,2}$

'Department of Biomedical \& Clinical Sciences 'L Sacco', University of Milan, via GB Grassi, 74, 20157 Milan, Italy ${ }^{2}$ Surgery Department, Breast Unit, ICS Maugeri S. p. A. SB, 27100 Pavia, Italy

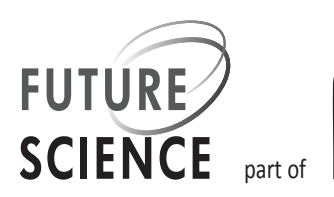


poly- $\gamma$-glutamic acid and their derivatives, or synthetic polymers like poly(lactide-co-glycolide), pluronic/ poly(lactic acid), poly(E-caprolactone) and L-lysine/Lleucine-based poly(ester amide) [7]. Gastric degradation has been avoided by coating with $\mathrm{pH}$-sensitive materials able to protect insulin in the stomach and to release it in the GI tract to promote bowel absorption. Moreover, enzyme inhibitors have been used to protect insulin from the activity of pepsin and other proteases, while mucoadhesive materials have been employed to increase the paracellular absorption enhancing intestinal retention time [7]. Also the increase of the insulin uptake by transcellular route has been studied developing targeted nanoparticles against enterocytes, mucus layer and $\mathrm{M}$ cells, through the functionalization with lectins. Despite this, oral delivery of insulin remains an open challenge [7].

In this scenario, the development of systems able to allow the targeted delivery and release of insulin in the colon has gained increasing interest by researchers, since colonic release has several advantages. It results in longer transit time, prolonged localization of insulin on the gut mucosa, exposition to lower levels of proteases or mucosal P-glycoprotein and increased responsiveness to permeation enhancers in comparison to that observed in small intestine [8]. However, the development of an efficient system for colonic release of insulin is stiff [9-13]. Although the potential of colloidal nanoparticles in increasing insulin absorption throughout the colon has been proved by some ex vivo and in vivo studies, they are limited by their inability to ensure nanoparticle safe transit through the stomach and small intestine [14,15]. An interesting approach that combines nanotechnology with drug formulation technology has been recently proposed using a pellet, which hosts insulin-loaded polyethylenimine nanoparticles [4]. The pellet is constituted by threelayer release technology platform, which combines an outer gastroresistant layer with a flexible film of a neutral polymethacrylate Eudragit ${ }^{\circledR} \mathrm{NE}$ and sodium starch glycolate Explotab $^{\circledR}$, applied to a hydroxypropyl methylcellulose coating [16-18]. Only the combination

\section{References}

1 Pettus J, Cavaiola TS, Tamborlane VW, Edelman S. The past, present, and future of basal insulins. Diabetes Metab. Res. Rev. 32, 478-496 (2016).

2 Polonsky KS, Given BD, Van Cauter E. Twenty-four-hour profiles and pulsatile patterns of insulin secretion in normal and obese subjects. J. Clin. Invest. 81(2), 442-448 (1998).

3 Hancu N, Czupryniak L, Genestin E, Sourij H. A panEuropean and Canadian prospective survey to evaluate patient satisfaction with the SoloSTAR insulin injection of this three-layer technology with PEI nanoformulation is able to mediate colonic release of insulin obtaining a long-lasting hypoglycemic effect [4]. These results underline the crucial role of the combination of threelayer technology and nanoparticle-mediated insulin delivery and pave a new way to obtain therapeutic efficacy for oral insulin.

These findings support the idea that the release of nanoformulated insulin in the colon holds a significant potential in clinical practice and could be a real solution to the hurdles in insulin oral delivery.

Indeed, insulin release in the colon should allow the restoration of a physiological delivery of insulin into the portal vein, with a proper liver metabolism, avoiding the fluctuation of blood glucose levels to obtain better control of glycemia, which is the major limitation registered in clinical and preclinical trials with oral insulin [19]. Moreover, this colon absorption of insulin mimics the physiological insulin circulation in the portal vein and the extraction by the liver, allowing this formulation to obviate the drawbacks of insulin administered subcutaneously, such as systemic hyperinsulinemia, local lipoatrophy, weight gain, atherogenesis and enhanced lipogenesis. Further research is mandatory to clarify the efficacy of this approach in postprandial glycemic control and the influence of this release of insulin on other metabolic and diabetes indicators. However, this new strategy for oral insulin that targets the colon seems to pave a new way in oral delivery of insulin.

\section{Financial \& competing interests disclosure}

The research was supported by the Fondazione Regionale per la Ricerca Biomedica (NANODRUG platform project to $F$ Corsi), and the Regione Lombardia and Fondazione Cariplo (2016-0919 to S Mazzucchelli). The authors have no other relevant affiliations or financial involvement with any organization or entity with a financial interest in or financial conflict with the subject matter or materials discussed in the manuscript apart from those disclosed.

No writing assistance was utilized in the production of this manuscript.

device in Type 1 and Type 2 diabetes. J. Diabetes Sci. Technol. 5(5), 1224-1234 (2011).

4 Salvioni L, Fiandra L, Del Curto MD et al. Oral delivery of insulin via polyethylene imine-based nanoparticles for colonic release allows glycemic control in diabetic rats. Pharmacological Res. 110, 122-130 (2016).

5 Sousa F, Castro P, Fonte P, Sarmento B. How to overcome the limitations of current insulin administration with new non-invasive delivery systems. Ther. Deliv. 6, 83-94 (2015).

6 Verma A, Kumar N, Malviya R et al. Emerging trends in noninvasive insulin delivery. J. Pharm. 2014, 1-9 (2014). 

YY, Xiong XY, Tian Y, Li ZL, Gong YC, Li YP. A review of biodegradable polymeric systems for oral insulin delivery. Drug Deliv. 23(6), 1882-1891 (2016).

Chen S, Guo F, Deng T et al. Eudragit S100-coated chitosan nanoparticles co-loading tat for enhanced oral colon absorption of insulin. AAPS PharmSciTech doi:10.1208/ s12249-016-0594-z (2016) (Epub ahead of print).

9 Maroni A, Zema L, Del Curto MD, Foppoli A, Gazzaniga A. Oral colon delivery of insulin with the aid of functional adjuvants. Adv. Drug. Deliv. Rev. 64, 540-556 (2012).

10 Patel MM. Colon targeting: an emerging frontier for oral insulin delivery. Exp. Opin. Drug Deliv. 10, 731-739 (2013).

11 Gazzaniga A, Maroni A, Sangalli ME, Zema L. Timecontrolled oral delivery systems for colon targeting. Exp. Opin. Drug Deliv. 3, 583-597 (2006).

12 Haupt $S$, Rubinstein A. The colon as a possible target for orally administered peptide and protein drugs. Crit. Rev. Ther. Drug Carr. Syst., 19, 499-551 (2002).

13 Bourgeois S, Harvey R, Fattal E. Polymer colon drug delivery systems and their application to peptides proteins, and nucleic acids. Am. J. Drug Deliv. 3, 171-204 (2005).
14 Bayat A, Dorkoosh FA, Dehpour AR et al. Nanoparticles of quaternized chitosan derivatives as a carrier for colon delivery of insulin: ex vivo and in vivo studies. Int. J. Pharm. 356, 259-266 (2008).

15 Cheng K, Lim LY. Insulin-loaded calcium pectinate nanoparticles: effects of pectin molecular weight and formulation pH. Drug. Dev. Ind. Pharm. 30, 359-367 (2004).

16 Palugan L, Cerea M, Zema L, Gazzaniga A, Maroni A. Coated pellets for oral colon delivery. J. Drug Deliv. Sci. Technol. 25, 1-15 (2015).

17 Maroni A, Del Curto MD, Cerea M, Zema L, Foppoli A, Gazzaniga A. Polymeric coatings for a multiple-unit pulsatile delivery system: preliminary study on free and applied films. Int. J. Pharm. 440, 256-263 (2013).

18 Del Curto MD, Palugan L, Foppoli A, Zema L, Gazzaniga A, Maroni A. Erodible time-dependent colon delivery systems with improved efficiency in delaying the onset of drug release. J. Pharm. Sci. 103, 3585-3593 (2014).

19 Zijlstra E, Heinemann L, Plum-Mörschel L. Oral insulin reloaded: a structured approach. J. Diabet. Sci. Technol. 8, 458-465 (2014). 
